\title{
Numerical investigation and Comparative analysis of tunable graphene-gold metasurface based polarizer structures for infrared frequency
}

\section{Vishal Sorathiya}

Marwadi University

shobhit patel ( $\square$ shobhitkumar.patel@marwadieducation.edu.in )

Marwadi University https://orcid.org/0000-0002-0117-2440

\section{Research Article}

Keywords: Polarizer, Graphene, Metasurface

Posted Date: October 29th, 2021

DOI: https://doi.org/10.21203/rs.3.rs-1031884/v1

License: (c) (1) This work is licensed under a Creative Commons Attribution 4.0 International License.

Read Full License 


\title{
Numerical investigation and Comparative analysis of tunable graphene-gold metasurface based polarizer structures for infrared frequency
}

\author{
Vishal Sorathiya ${ }^{1,3}$, Shobhit K Patel ${ }^{2,3 \#}$ \\ ${ }^{1}$ Department of Information and Communication Technology, Marwadi University, Gujarat, \\ Rajkot, 360003 India \\ ${ }^{2}$ Department of Computer Engineering, Marwadi University, Gujarat, Rajkot, 360003 India \\ ${ }^{3}$ Department of Electronics and Communication, Marwadi University, Gujarat, Rajkot, 360003 \\ India \\ ${ }^{\#}$ Corresponding Author: shobhitkumar.patel@marwadieducation.edu.in
}

\begin{abstract}
:
We present the comparative analysis of tunable graphene-based metasurface polarizer structure $\mathrm{THz}$ frequency range. This polarizer structures have been numerically investigated over the 10 $\mathrm{THz}$ to $25 \mathrm{THz}$ of the frequency range. Reflectance co-efficient, phase variation, and phase difference parameters have been investigated to identify the behavior of polarization effect over the range of $10 \mathrm{THz}$ to $25 \mathrm{THz}$ of the frequency. The proposed polarizer has been also investigated for the different shapes of the top gold resonator structure. The proposed structure is tunable for the range of $0.1 \mathrm{eV}$ to $0.9 \mathrm{eV}$ of the graphene Fermi energy. The proposed structure also works on the wide range of the input incident wave of the $\mathrm{X}$ and $\mathrm{Y}$ polarization. This structure having small, compact tunable design and it can be used as a basic building block in the large $\mathrm{THz}$ circuits and structures.
\end{abstract}

Keywords: Polarizer; Graphene; Metasurface.

\section{Introduction:}

Two elements control an optical device's electromagnetic response: the material and structure, from simple lenses and mirrors to state-of-the-art metasurface alternatives. Conventional optics beam engineering depends on different refractive index $n$ contributions with interface profiles resulting in minimal flexibility and bulky size. Relentless demand for nano-fabrication results in the minimization of developing meta-faces to provide an excellent medium for transforming a beam over a meta-atoms thin layer[1], [2]. Profound structures of meta-atoms, couple the light with a heavy field position to change the effective material property locally as an effective refractive index which is very distinct from that of the natural material. Beam shaping from metaatoms with an ultrathin layer of defined thickness $d$ is possible with the configuration of spatial effective refractive index. Metasurfaces considerably decrease the dimensions of the photonics devices with identical or novel features usable, such as metals with standardized numerical openings, polarization holography [3], [4], profile planner[5], spin-angular momentum coupling [6], [7], and multi-function synthesis [8], [9], just to mention but a few. In various applications like dynamic adaptive optics, wavefront architecture, and pixel detection, active or reconfigurable responses are very attractive and can be enabled by the use of usable materials in dynamic modulation-based metasurfaces. Graphene is a six carbon atoms formed into a honeycomb lattice arrangement[10] with a single-layered structure. Graphene is an excellent material that will provide excellent optical and electrical properties because its low-carrier-density and Fermi-level related can be significantly controlled by external DC gate bias. Structured graphene by different fabrication techniques or graphene integration with other passive metasurfaces is an important 
approach for optimizing dynamic beam-engineering modulation [11]. The material and structure promote each other with graphene- metasurfaces in order to improve the light interaction with the ultrathin atomic layer and to modulate the reaction of meta-atoms by adjusting the tunable material quality of graphene[12]. Metasurfaces based on graphene offer a number of benefits over those based on other active materials and tuning processes [13], electrically tuned liquid crystals [14], and mechanically deformed elastics [15]. At micro and millimeter-wave wavelengths, the biperiodic nature of the metal was long used for the achievement of electromagnetic properties that cannot be achieved with regular multi-layered structures. FSS, spectrum filters (used in radar technology) [16] are perhaps the most common applications for such periodic screens. The dynamic management of different FSSs with fixed frequency responses remains a problem despite excellent modelling, application, and usage. The process that enables the FSS output to be dynamically regulated usually sets very difficult limits on the achievable. Due to the abovementioned technical factors, however, it is much more difficult to obtain such characteristics in infrared regimes in the microwaves scheme to have the dynamic frequency response[17]. However, such characteristics in the Terahertz and Infrared regimes are recorded. Graphs are normally compliant with silicon photonics and planar metasurfaces as the thinnest active material. It's possible to tune electrically at extremely high velocities, ranging from [18] to $\mathrm{GHz}$ [19]. Moreover, the material properties of graphene can be modulated electrically from subterahertz (sub-THz) to near-infrared in an incredibly large range (near-IR). Interestingly, when it comes to meta-device adjusting features, the focus is on the real portion at sub-THz and the imaginary part at higher frequencies, which are both contributing to various meta-device adjustability characteristics. Graphene metasurfaces evolve from the modulation of intensity. In $2011, \mathrm{THz}$ wave modulation strength was recorded in the graphene micro-ribbon array accompanied by several modulators [20]-[22], tunable metasurface absorbers [23][24][25]. and modulators of spatial light [26]. In recent years, besides amplitude modulation, growing emphasis has centred on the modulation process, with various theoretical ideas and many experimental breakthroughs, for dynamic wave front formation.

In this paper, we have designed a tunable graphene-gold-based polarizer structure for the far-infrared frequency spectrum. We have investigated various physical parameters for different input polarization conditions. We have investigated the behavior of the polarizer for the different gold resonator geometry. The manuscript has been organized into three sections of polarizer structure design, graphene, and effect of the conductivity and results and discussion. The proposed graphene-based polarizer structures are also compared in terms of the reflectance coefficient, polarization, and bandwidth of reflectance.

\section{Graphene-based Tunable Polarizer structure:}

A graphene-based tunable infrared polarizer structure has been shown in Fig. 1. The threedimensional view of the polarizer structure is shown in Fig. 1 (a). The proposed structure has been investigated for the different resonator conditions of the gold geometry as shown in Fig. 1(b-e). The basic geometries are split ring resonator (SRR), rectangular resonator (RR), triangular split ring resonator (TSRR), squared split ring resonator (SSRR) considered for the polarizer design. The dimensions of the structures has defined as follows: $\mathrm{W}=7.6 \mu \mathrm{m}, \mathrm{L}=7.6 \mu \mathrm{m}, \mathrm{C}_{1}=1.5 \mu \mathrm{m}, \mathrm{C}_{\mathrm{w}}$ $=1.5 \mu \mathrm{m}, \mathrm{S}_{1}=6.5 \mu \mathrm{m}, \mathrm{S}_{\mathrm{w}}=5 \mu \mathrm{m}, \mathrm{g}_{\mathrm{p}}=0.75 \mu \mathrm{m}, \mathrm{g}_{\mathrm{h}}=0.2 \mu \mathrm{m}, \mathrm{g}_{\mathrm{r}}=0.375 \mu \mathrm{m}, \mathrm{S}_{\mathrm{h}}=1.5 \mu \mathrm{m}, \mathrm{r}_{\mathrm{i}}=1.5$ $\mu \mathrm{m}, \mathrm{r}_{\mathrm{w}}=3 \mu \mathrm{m}, \mathrm{t}_{\mathrm{w}}=4.47 \mu \mathrm{m}, \mathrm{g}_{1}=3 \mu \mathrm{m}, \mathrm{g}_{\mathrm{w}}=3 \mu \mathrm{m}$. The Finite Element Method (FEM) was used to quantitatively analyse the proposed structure. Fig. 1 (a) shows the incident wave launching from 
the structure's top in a $\mathrm{Z}$ direction. Periodic boundary conditions in the $\mathrm{X}$ and $\mathrm{Y}$ directions have been used to evaluate the suggested structure.

\section{Graphene modeling and conductivity equation:}

The conductivity values of the single-layer graphene can be expressed by the Kubo formula [27]. The equation of the graphene conductivity model and permittivity is shown in Eq. (1-2). Graphene in the proposed polarizer structure has been defined as a surface conductivity model as a singlelayer structure. The surface current destiny values on both of the axis have defined as $J_{x}=$ $E_{x} \sigma_{s}$ and $J_{y}=E_{y} \sigma_{s}$.

$$
\begin{gathered}
\varepsilon(\omega)=1+\frac{\sigma_{s}}{\varepsilon_{0} \omega \Delta} \\
\sigma_{s}=\frac{-j e^{2} k_{B} T}{\pi \hbar^{2}(\omega-j 2 \Gamma)}\left(\frac{E_{f}}{k_{B} T}+2 \ln \left(e^{-\frac{E_{f}}{k_{B} T}}+1\right)\right)+\frac{-j e^{2}}{4 \pi \hbar} \ln \left(\frac{2\left|E_{f}\right|-(\omega-j 2 \Gamma) \hbar}{2\left|E_{f}\right|+(\omega-j 2 \Gamma) \hbar}\right)
\end{gathered}
$$

Parameter values of the above equation have been shown in Table 1. The graphene Fermi potential as a function of gate bias voltage has defined as $E_{f}=\hbar v_{F} \sqrt{\pi C V_{b g}}$. Here $\mathrm{C}=\varepsilon_{0} \varepsilon_{\mathrm{d}} / \mathrm{S}_{h}$ is Electro statistic capacitance per unit area. Where, $\varepsilon_{0}$ defined as permittivity of free space, $\varepsilon_{\mathrm{d}}(2.25)$ define as permittivity of silica material. $S_{h}(1.5 \mu \mathrm{m})$ is the thickness of the silica layer. Complex valued results will be produced using the graphene conductivity equation Resistive and reactive behaviour are both affected by this equation. Under the calculation of the graphene sheet in FEM simulation, Graphene has a thickness of $0.39 \mathrm{~nm}$.

MBE [28], CVD [29] and Cleavage [30] techniques are the basic methods of fabricating the two-dimensional and graphene-like single layer material. Graphene's top layer may be formed into the complex structure by using nano spheric lithography [31] and nano-plasmonics plasmonics lithography [32]. There are lithography methods for plasmonics device manufacturing that allow high quality graphene composites with complicated nano and microstructures, as shown in [32]. Graphene transfer printing with gold film may be used to transfer gold structure onto the graphene layer [33]. It is shown an experimental procedure for producing a gold surface with a design [32].

\section{Result and Discussion:}

The split ring resonator geometries offer different resonating bands as the capacitive and inductive entity introduces for the different metamaterial geometries. The gap between the two ends of the split ring generates the capacitive effect. The area and the structure of the different geometries generate the different dipole moments over the $\mathrm{THz}$ region. The proposed graphene gold-based polarizer structure has been simulated and results are numerically investigated for the range of 10 to $25 \mathrm{~T} \mathrm{~Hz}$ of the far-infrared frequency range. We have investigated the behavior of the polarizer for the different gold resonator conditions. The reflectance coefficient is defined as $R_{i j}=\left|E_{j}^{\text {Reflec }} / E_{i}^{\text {Inc }}\right|(i, j=x, y)$, where $E_{j}^{\text {Reflec }}(j=x, y)$ is the $\mathrm{x}$ or $\mathrm{y}$ component of the reflected wave and $E_{j}^{I n c}(j=x, y)$ is the $\mathrm{x}$ or y component of the incident wave [33]. Variation in the reflectance co-efficient of all structures has been shown in Fig. 2. Fig 2 (a) and Fig. 2(b) shows the variation in the $R_{x x}$ and $R_{y y}$ for the RR geometry polarizer. Similarly, $R_{x x}$ and $R_{y y}$ for the other gold, geometry condition has been shown in Fig. $2(\mathrm{c}-\mathrm{g})$. The results of $R_{x x}$ and $R_{y y}$ were 
also computed for various graphene sheet fermi energy levels. The contour plot of the reflection co-efficient shows that the proposed polarizer structures have been tunable for the different Fermi energy values of the graphene sheet. It is also observed that the reflectance response of all the structures is different for the same range of frequency. It is observed that $R_{x x}$ is shows the wide band of the reflectance over the range of $10 \mathrm{THz}$ to $25 \mathrm{THz}$ in all the structures. On the other side, values of $R_{y y}$ is higher for the large $\mathrm{THz}$ frequency and fermi energy of graphene sheet. The phase is defined as $\Phi_{i j}=\arg \left(E_{j}^{\text {Reflec }} / E_{i}^{\text {Inc }}\right)(i, j=x, y)$.

The phase variation for the $R_{x x}$ and $R_{y y}$ are shown in Fig. 3. The phase variation ( $\Phi_{x x}, \Phi_{y y}$ ) and phase difference $(\Delta \Phi)$ for the RR has been shown in Fig. 3 (b). The related reflection coefficient for the same resonating structure has been shown in Fig. 3(a). Similarly, the Reflectance co-efficient and the phase variation for the different resonating structures have been shown in Fig. 3 (c-h). Fig. 3 shows that for higher values of the reflectance the phase variation increases. It related that for the rise of the reflectance value the phase variation is also higher. It is also observed that for SSRR, TSRR, and SSR phase difference $\Delta \Phi$ is large as compared to RR structure. It is also observed that phase differences in SSRR, TSRR, and SSR higher for the lower values of reflectance. Fig. 3 has been derived by considering the graphene Fermi energy of 0.7 $\mathrm{eV}$. We have also looked at how phase variation responds to graphene sheet Fermi energy variations. Variation of the $\Phi_{x x}$ for multiple values of $E_{f}$ and frequency has been shown in Fig. 4 for different resonating structures. Similarly, variation in $\Phi_{y y}$ and $\Delta \Phi$ for the multiple values of $E_{f}$ and frequency has been shown in Fig. 5 and Fig. 6. It is found that the values of $\Phi_{y y}, \Phi_{y y}$ and $\Delta \Phi$ regulates itself depending on the frequency and fermi potential value. The transmission resonance and phase difference are shifted due to the variation in the length $\left(\mathrm{S}_{\mathrm{l}}\right)$ and width $\left(\mathrm{S}_{\mathrm{w}}\right)$ of the graphene sheet in a different mode of the incident polarization in X or Y directions. This asymmetry generates different resonance peaks at different points. To achieve the bigger difference in wavelength shift can be possible by making the difference larger between $S_{1}$ and $S_{w}$. The resonance frequency of the graphene depends on the length of the structure as well as width by considering the function $f_{r} \alpha \sqrt{E_{f} / L}$ [34]. The resonance of the specific frequency can be generated by the dipole moment created by the graphene sheet and the gold resonator structure. Shape of top resonator structure has an effect on resonance frequency, as shown by the results of this experiment. The reflectance, PCR, phase variation with co polarization and cross-polarization shows the different operation region for a different gold resonator structure. The effect of the area of resonator, edges, shape, and gap between split ring offers the different resonating dipole moments. This will result in the different operating regions of the polarizer. This study will show the numerically investigated results to identify the operating band using the shape of the gold resonator.

For all types of resonator constructions, wide-angle incident polarisation stability of the reflectance coefficient is illustrated in Fig. 7. It has been determined that the suggested construction is effective at incidence angles ranging from $0^{\circ}$ to $80^{\circ}$. The wide-angle response shows the constant reflectance response on the multiple resonating peaks. We have also compared the proposed structure with the previously published structure and design as shown in Table 3 in terms of dimensions, angular stability, reflectance amplitude, and forming materials.

\section{Conclusion:}

In conclusion, an ultrathin tunable graphene-based infrared polarizer has been demonstrated. It is also demonstrated the performance of the polarizer structures for the different geometry of the top 
resonating structures. Compare to the previously presented polarizer structure, the presented structure is easy to verify experimentally. The polarization variation and the phase difference of the structure have been identifying for the different incident values of the polarization. The polarizer structure has been also verified for the wide-angle of the incident wave to check the stability of polarization conversion. The graphene sheet's potential (Fermi energy) may be adjusted by changing the polarizer configuration. From the comparative study, it can be shown that the polarizer behaves differently in each resonating structure. A large optoelectronics system for infrared frequencies may be constructed using the suggested polarizer structure because of its simple and compact construction.

Data Availability: My manuscript has no associated data.

Conflict of Interest: The authors declare that they have no conflict of interest.

\section{Reference:}

[1] Q. He, S. Sun, S. Xiao, and L. Zhou, "High-Efficiency Metasurfaces: Principles, Realizations, and Applications," Adv. Opt. Mater., vol. 6, no. 19, p. 1800415, Oct. 2018, doi: 10.1002/adom.201800415.

[2] S. M. Kamali, E. Arbabi, A. Arbabi, and A. Faraon, "A review of dielectric optical metasurfaces for wavefront control," Nanophotonics, vol. 7, no. 6, pp. 1041-1068, Jun. 2018, doi: 10.1515/nanoph-2017-0129.

[3] W. T. Chen et al., "High-efficiency broadband meta-hologram with polarizationcontrolled dual images," Nano Lett., vol. 14, no. 1, pp. 225-230, Jan. 2014, doi: $10.1021 / \mathrm{n} 1403811 \mathrm{~d}$.

[4] D. Wen et al., "Helicity multiplexed broadband metasurface holograms," Nat. Commun., vol. 6, no. 1, pp. 1-7, Sep. 2015, doi: 10.1038/ncomms9241.

[5] V. V. Kotlyar et al., "Thin high numerical aperture metalens," Opt. Express, vol. 25, no. 7, p. 8158, Apr. 2017, doi: 10.1364/OE.25.008158.

[6] F. Zhang et al., "All-Dielectric Metasurfaces for Simultaneous Giant Circular Asymmetric Transmission and Wavefront Shaping Based on Asymmetric Photonic Spin-Orbit Interactions," Adv. Funct. Mater., vol. 27, no. 47, p. 1704295, Dec. 2017, doi: 10.1002/adfm.201704295.

[7] G. Li et al., "Nonlinear Metasurface for Simultaneous Control of Spin and Orbital Angular Momentum in Second Harmonic Generation," Nano Lett., vol. 17, no. 12, pp. 7974-7979, Dec. 2017, doi: 10.1021/acs.nanolett.7b04451.

[8] Z.-L. Deng, Y. Cao, X. Li, and G. P. Wang, "Multifunctional metasurface: from extraordinary optical transmission to extraordinary optical diffraction in a single structure," Photonics Res., vol. 6, no. 5, p. 443, May 2018, doi: 10.1364/PRJ.6.000443.

[9] A. Forouzmand, M. M. Salary, S. Inampudi, and H. Mosallaei, "A Tunable Multigate Indium-Tin-Oxide-Assisted All-Dielectric Metasurface," Adv. Opt. Mater., vol. 6, no. 7, p. 1701275, Apr. 2018, doi: 10.1002/adom.201701275.

[10] A. K. Geim and K. S. Novoselov, "The rise of graphene," Nat. Mater., vol. 6, no. 3, pp. 183-191, Mar. 2007, doi: 10.1038/nmat1849.

[11] J. Cheng, F. Fan, and S. Chang, "Recent progress on graphene-functionalized metasurfaces for tunable phase and polarization control," Nanomaterials, vol. 9, no. 3, 2019, doi: 10.3390/nano9030398. 
[12] K. S. Novoselov, V. I. Fal'Ko, L. Colombo, P. R. Gellert, M. G. Schwab, and K. Kim, “A roadmap for graphene," Nature, vol. 490, no. 7419. Nature Publishing Group, pp. 192200, Oct. 2012, doi: 10.1038/nature11458.

[13] S. K. Patel, J. Parmar, R. B. Zakaria, S. A., T. K. Nguyen, and V. Dhasarathan, "Sensitivity Analysis of Metasurface Array-Based Refractive Index Biosensors," IEEE Sens. J., 2021, doi: 10.1109/JSEN.2020.3017938.

[14] S. Savo, D. Shrekenhamer, and W. J. Padilla, "Liquid crystal metamaterial absorber spatial light modulator for THz applications," Adv. Opt. Mater., vol. 2, no. 3, pp. 275-279, Mar. 2014, doi: 10.1002/adom.201300384.

[15] C. Zaichun, M. Rahmani, G. Yandong, C. T. Chong, and H. Minghui, "Realization of Variable Three-Dimensional Terahertz Metamaterial Tubes for Passive Resonance Tunability," Adv. Mater., vol. 24, no. 23, pp. OP143-OP147, Jun. 2012, doi: 10.1002/adma.201104575.

[16] R. Anwar, L. Mao, and H. Ning, "Frequency Selective Surfaces: A Review," Appl. Sci., vol. 8, no. 9, p. 1689, Sep. 2018, doi: 10.3390/app8091689.

[17] B. Schoenlinner, A. Abbaspour-Tamijani, L. C. Kempel, and G. M. Rebeiz, "Switchable low-loss RF MEMS Ka-band frequency-selective surface," in IEEE Transactions on Microwave Theory and Techniques, Nov. 2004, vol. 52, no. 11, pp. 2474-2481, doi: 10.1109/TMTT.2004.837148.

[18] K.Sumathi, S. Lavadiya, P. Z. Yin, J. Parmar, and S. K. Patel, "High gain multiband and frequency reconfigurable metamaterial superstrate microstrip patch antenna for $\mathrm{C} / \mathrm{X} / \mathrm{Ku}-$ band wireless network applications," Wirel. Networks, 2021, doi: 10.1007/s11276-02102567-5.

[19] M. Kamran Shereen, M. I. Khattak, and G. Witjaksono, “A brief review of frequency, radiation pattern, polarization, and compound reconfigurable antennas for $5 \mathrm{G}$ applications," Journal of Computational Electronics. 2019, doi: 10.1007/s10825-01901336-0.

[20] L. Thomas, V. Sorathiya, S. K. Patel, and T. Guo, "Graphene-based tunable near-infrared absorber," Microw. Opt. Technol. Lett., Jan. 2019, doi: 10.1002/mop.31712.

[21] V. Dave, V. Sorathiya, T. Guo, and S. K. Patel, "Graphene based tunable broadband farinfrared absorber," Superlattices Microstruct., vol. 124, pp. 113-120, Dec. 2018, doi: 10.1016/j.spmi.2018.10.013.

[22] S. K. Patel, M. Ladumor, V. Sorathiya, and T. Guo, "Graphene based tunable grating structure," Mater. Res. Express, vol. 6, no. 2, p. 025602, Nov. 2019, doi: 10.1088/20531591/aaea9a.

[23] S. Barimani, A. Amini, and I. Chaharmahali, "A four-bias three-layer graphene-based THz absorber," J. Comput. Electron., 2021, doi: 10.1007/s10825-021-01700-z.

[24] S. K. Patel, J. Parmar, V. Sorathiya, R. Zakaria, V. Dhasarathan, and T. K. Nguyen, "Graphene-Based Plasmonic Absorber For Biosensing Applications Using Gold Split Ring Resonator Metasurfaces,” J. Light. Technol., 2021, doi: 10.1109/JLT.2021.3069758.

[25] J. Parmar, S. K. Patel, D. Katrodiya, T. K. Nguyen, J. S. Skibina, and V. Dhasarathan, "Numerical investigation of gold metasurface based broadband near-infrared and nearvisible solar absorber," Phys. B Condens. Matter, 2020, doi: 10.1016/j.physb.2020.412248.

[26] N. Kakenov et al., "Graphene-enabled electrically controlled terahertz spatial light modulators," Opt. Lett., vol. 40, no. 9, p. 1984, May 2015, doi: 10.1364/ol.40.001984. 
[27] G. W. Hanson, "Dyadic Green's functions and guided surface waves for a surface conductivity model of graphene," J. Appl. Phys., vol. 103, no. 6, 2008, doi: 10.1063/1.2891452.

[28] E. Moreau et al., "Graphene growth by molecular beam epitaxy on the carbon-face of SiC," Appl. Phys. Lett., vol. 97, no. 24, p. 241907, Dec. 2010, doi: 10.1063/1.3526720.

[29] N. Petrone et al., "Chemical Vapor Deposition-Derived Graphene with Electrical Performance of Exfoliated Graphene," Nano Lett., vol. 12, no. 6, pp. 2751-2756, Jun. 2012, doi: 10.1021/n1204481s.

[30] K. S. Novoselov et al., "Two-dimensional atomic crystals," Proc. Natl. Acad. Sci., vol. 102, no. 30, pp. 10451-10453, Jul. 2005, doi: 10.1073/pnas.0502848102.

[31] M. C. Sherrott et al., "Experimental Demonstration of \&gt;230 2 Phase Modulation in Gate-Tunable Graphene-Gold Reconfigurable Mid-Infrared Metasurfaces," Nano Lett., vol. 17, no. 5, pp. 3027-3034, May 2017, doi: 10.1021/acs.nanolett.7b00359.

[32] T. Zou et al., "High-speed femtosecond laser plasmonic lithography and reduction of graphene oxide for anisotropic photoresponse," Light Sci. Appl., vol. 9, no. 1, 2020, doi: 10.1038/s41377-020-0311-2.

[33] H. Cheng, S. Chen, P. Yu, J. Li, L. Deng, and J. Tian, "Mid-infrared tunable optical polarization converter composed of asymmetric graphene nanocrosses," Opt. Lett., vol. 38, no. 9, p. 1567, May 2013, doi: 10.1364/OL.38.001567.

[34] J. Ding et al., "Mid-Infrared Tunable Dual-Frequency Cross Polarization Converters Using Graphene-Based L-Shaped Nanoslot Array," Plasmonics, vol. 10, no. 2, pp. 351356, Apr. 2015, doi: 10.1007/s1 1468-014-9816-y.

[35] J. Zhu, S. Li, L. Deng, C. Zhang, Y. Yang, and H. Zhu, "Broadband tunable terahertz polarization converter based on a sinusoidally-slotted graphene metamaterial," Opt. Mater. Express, vol. 8, no. 5, p. 1164, May 2018, doi: 10.1364/OME.8.001164.

[36] X. Yu, X. Gao, W. Qiao, L. Wen, and W. Yang, "Broadband Tunable Polarization Converter Realized by Graphene-Based Metamaterial," IEEE Photonics Technol. Lett., vol. 28, no. 21, pp. 2399-2402, 2016, doi: 10.1109/LPT.2016.2596843.

[37] Z. Hu, M. Aqeeli, X. Zhang, X. Huang, and A. Alburaikan, "Design of broadband and tunable terahertz absorbers based on graphene metasurface: equivalent circuit model approach," IET Microwaves, Antennas Propag., vol. 9, no. 4, pp. 307-312, 2015, doi: 10.1049/iet-map.2014.0152.

[38] S. Liu, H. Chen, and T. J. Cui, "A broadband terahertz absorber using multi-layer stacked bars," Appl. Phys. Lett., vol. 106, no. 15, pp. 1-6, 2015, doi: 10.1063/1.4918289.

[39] A. Fardoost, F. G. Vanani, A. Amirhosseini, and R. Safian, "Design of a Multilayer Graphene-Based Ultrawideband Terahertz Absorber," IEEE Trans. Nanotechnol., vol. 16, no. 1, pp. 68-74, 2017, doi: 10.1109/TNANO.2016.2627939.

[40] E. S. Torabi, A. Fallahi, and A. Yahaghi, "Evolutionary Optimization of Graphene-Metal Metasurfaces for Tunable Broadband Terahertz Absorption," IEEE Trans. Antennas Propag., vol. 65, no. 3, pp. 1464-1467, Mar. 2017, doi: 10.1109/TAP.2016.2647580.

[41] E. Gao et al., "Dynamically tunable dual plasmon-induced transparency and absorption based on a single-layer patterned graphene metamaterial," Opt. Express, vol. 27, no. 10, p. 13884, 2019, doi: 10.1364/oe.27.013884.

[42] G. Deng, J. Yang, and Z. Yin, "Broadband terahertz metamaterial absorber based on tantalum nitride," Appl. Opt., vol. 56, no. 9, p. 2449, 2017, doi: 10.1364/ao.56.002449.

[43] X. Liu, Q. Zhang, and X. Cui, "Ultra-broadband Polarization-Independent Wide-Angle 
THz Absorber Based on Plasmonic Resonances in Semiconductor Square Nut-Shaped Metamaterials," Plasmonics, vol. 12, no. 4, pp. 1137-1144, 2017, doi: 10.1007/s11468016-0368-1.

[44] C. Shi et al., "Compact Broadband Terahertz Perfect Absorber Based on MultiInterference and Diffraction Effects," IEEE Trans. Terahertz Sci. Technol., vol. 6, no. 1, pp. 40-44, 2016, doi: 10.1109/TTHZ.2015.2496313. 
Figure 1:

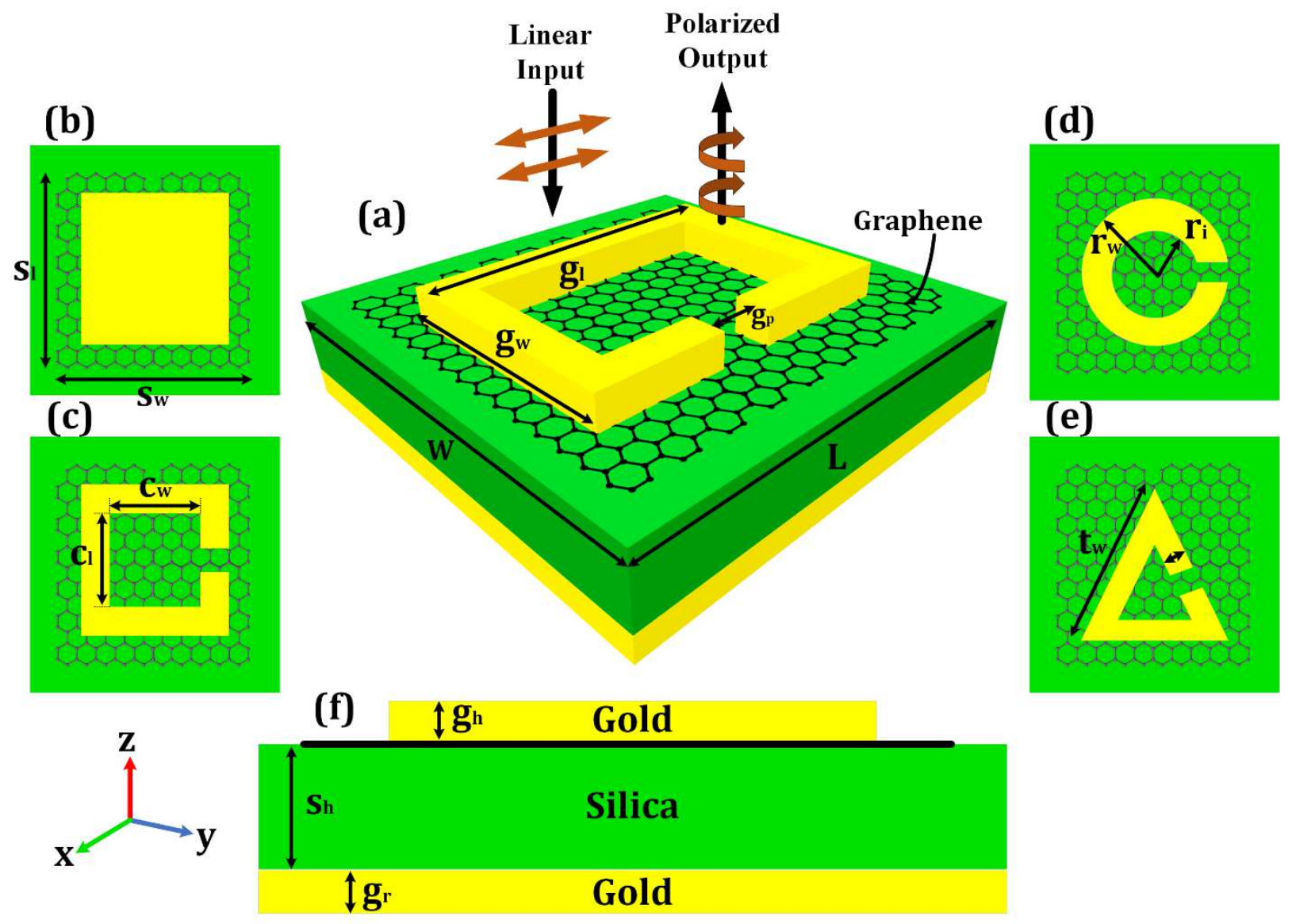

Schematic of the polarizer structure using graphene and gold resonator geometry. (a) Threedimensional (3-D) view of graphene gold based tunable polarizer structure. Top view of the different gold geometry such as (b) Rectangular resonators (RR), (c) Squared split ring resonator (SSRR), (d) Split ring resonator (SRR) and (e) triangular split ring resonator (TSRR). (f) The Side view of the polarizer structure. 
Figure 2:
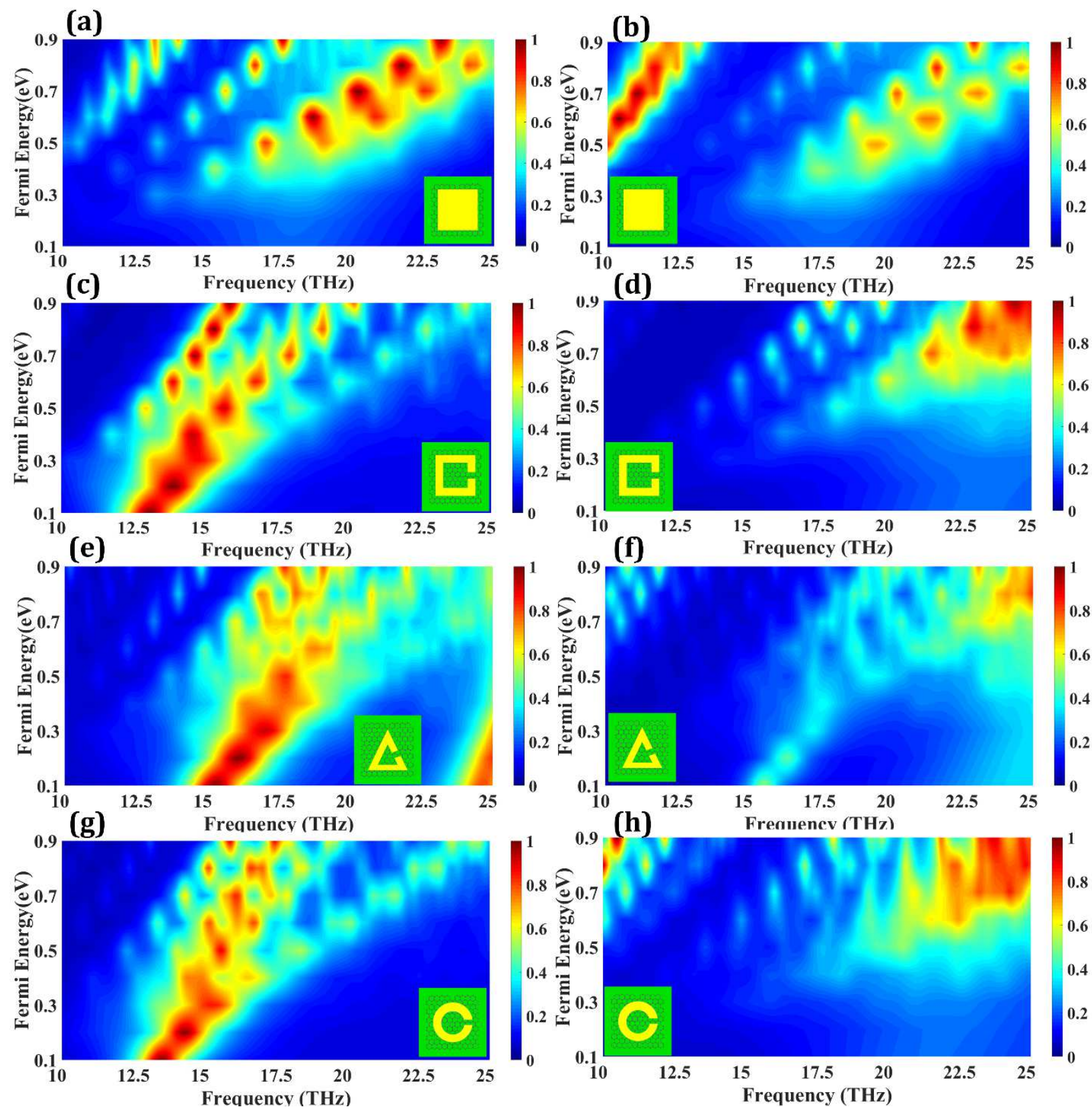

Calculated reflectance response $\left(R_{x x}\right.$ and $\left.R_{y y}\right)$ for the different values of graphene Fermi energy, frequency, and resonating structure for $\mathrm{X}$, Y polarized incident wave. (a) $R_{x x}$ and (b) $R_{y y}$ for RR geometry. (c) $R_{x x}$ and (d) $R_{y y}$ for SSRR geometry. (e) $R_{x x}$ and (f) $R_{y y}$ for TSRR geometry. (g) $R_{x x}$ and (h) $R_{y y}$ SRR geometry. The Colour bar shows the variation of reflectance amplitude from minimum to maximum.

Figure 3: 
(a)

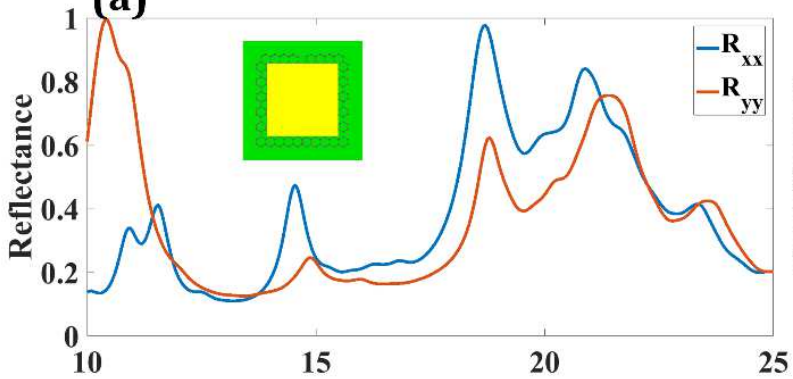

(c)
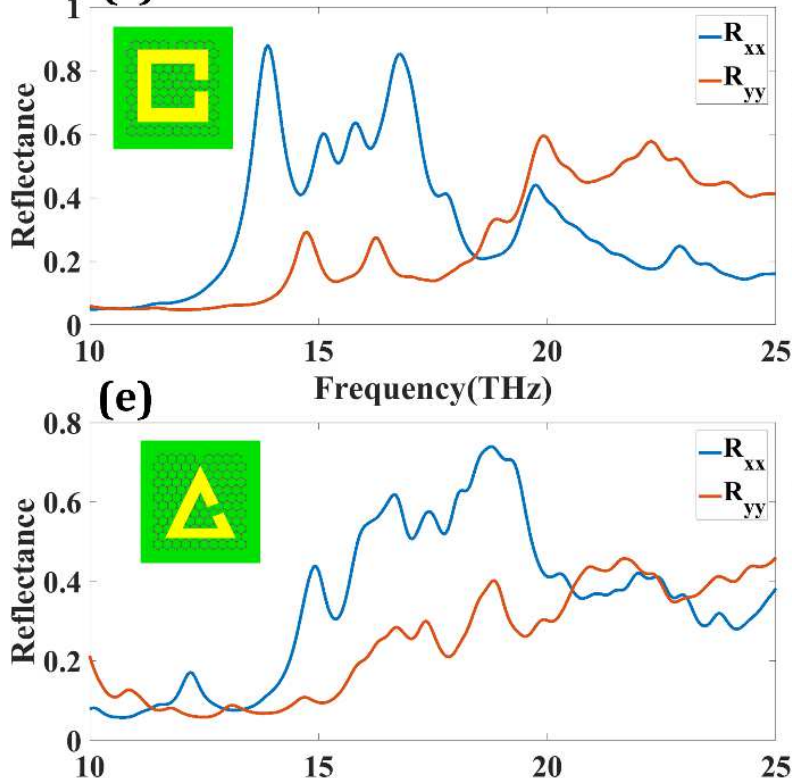

${ }_{0.8}$ (g) Frequency(THz)

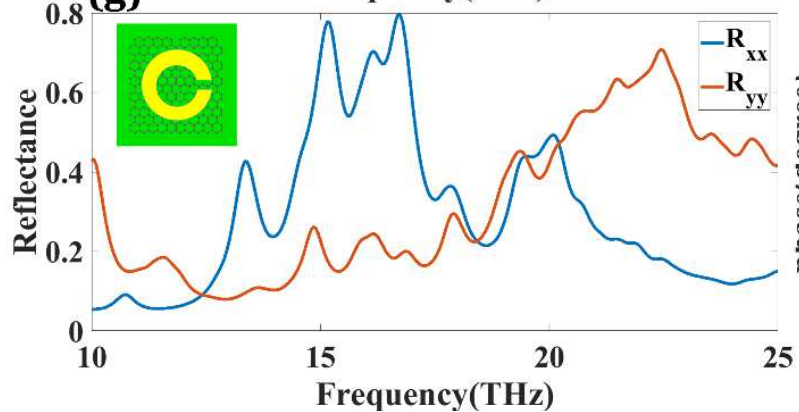

(b)

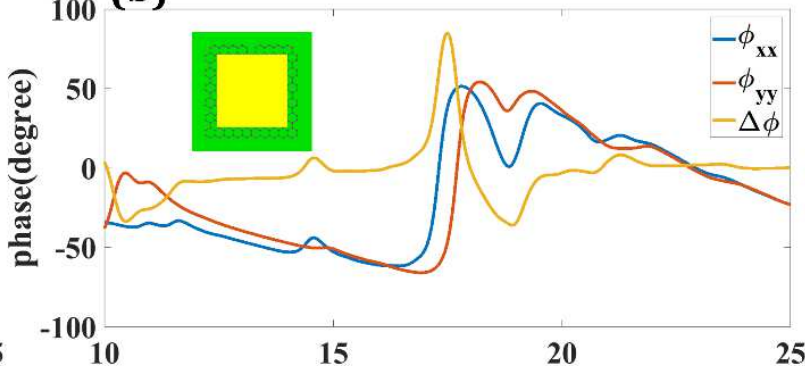

Frequency(THz)

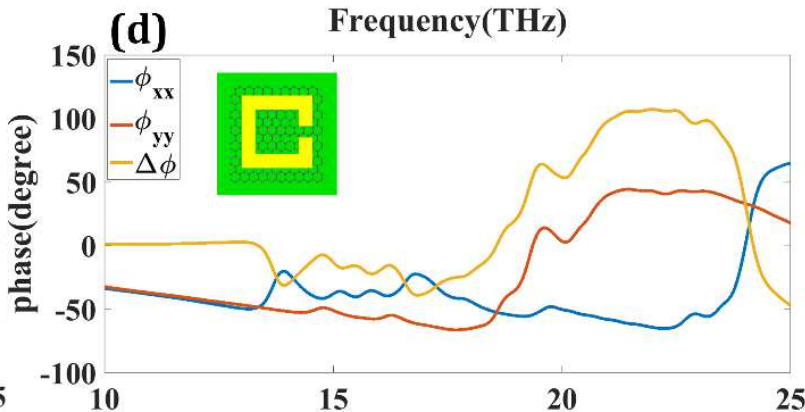

(f) Frequency(THz)

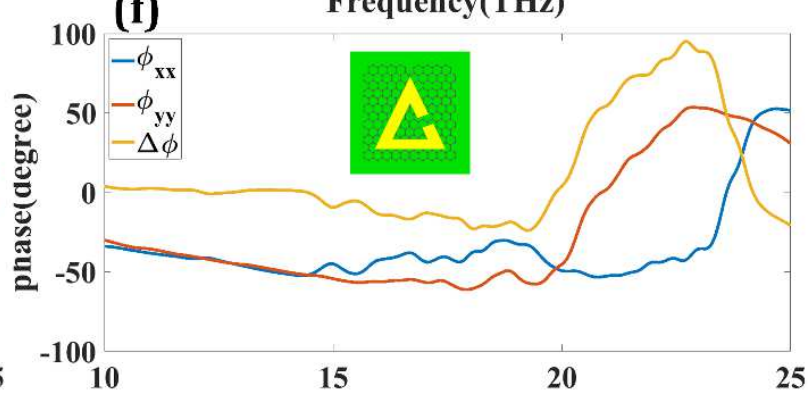

(h)

Frequency(THz)

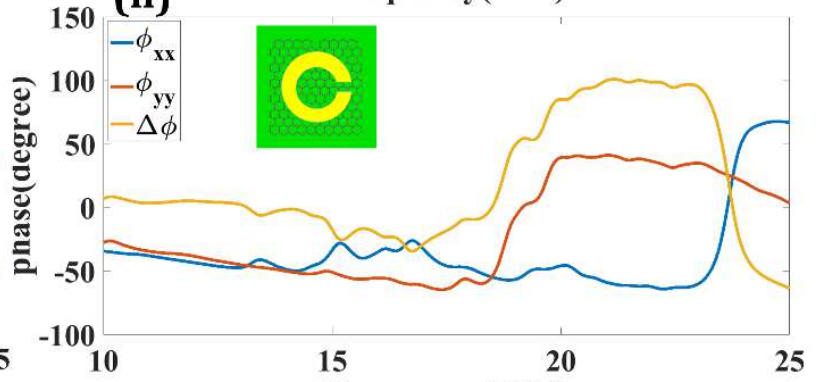

Calculated reflectance response $\left(R_{x x}\right.$ and $\left.R_{y y}\right)$, phase variation response $\left(\Phi_{x x}\right.$ and $\left.\Phi_{y y}\right)$ and phase difference $\Delta \Phi$ for the different resonator structures. $R_{x x}$ and $R_{y y}$ for the (a) RR, (c) SSRR, (e) TSRR and (g) SRR structures. $\Phi_{x x}, \Phi_{y y}$ and $\Delta \Phi$ for the (b) RR, (d) SSRR, (f) TSRR, and (h) SRR structures. 
Figure 4:

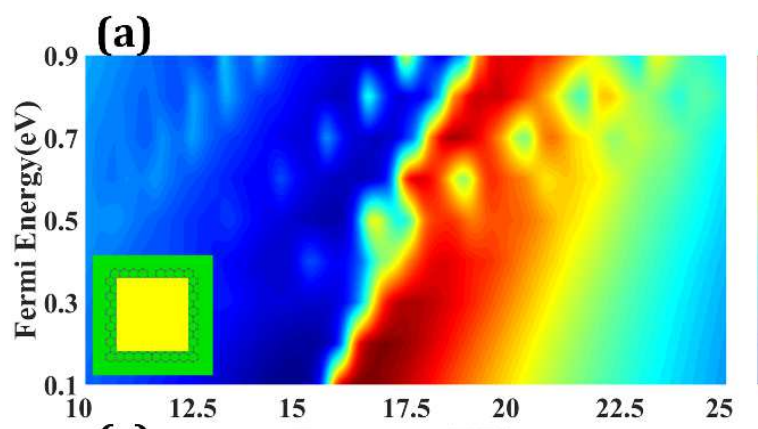

(c) Frequency (THz)

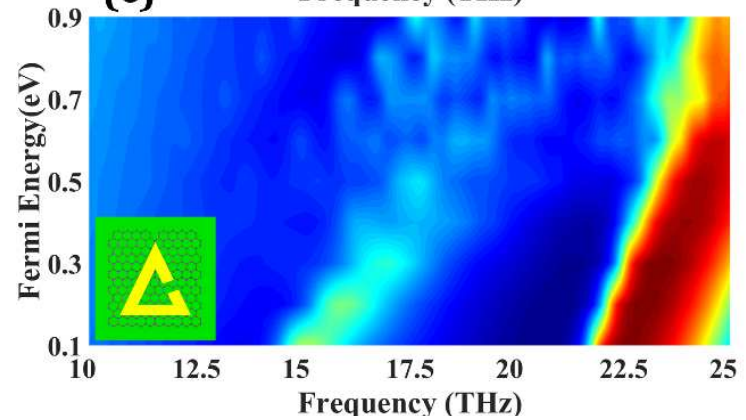

(b)

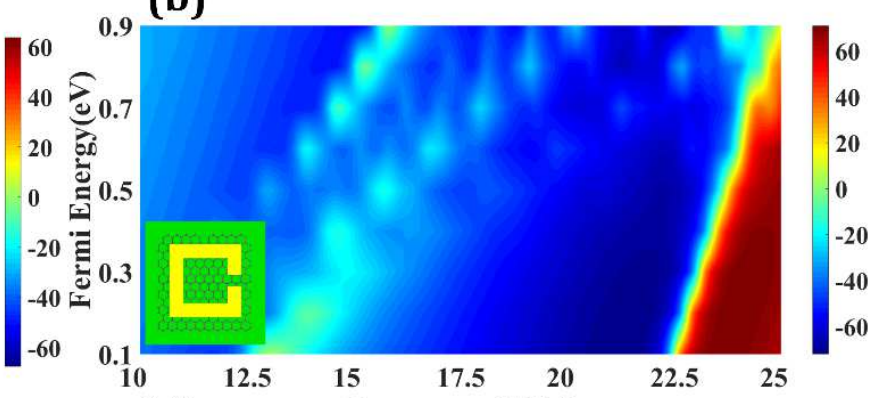

(d)

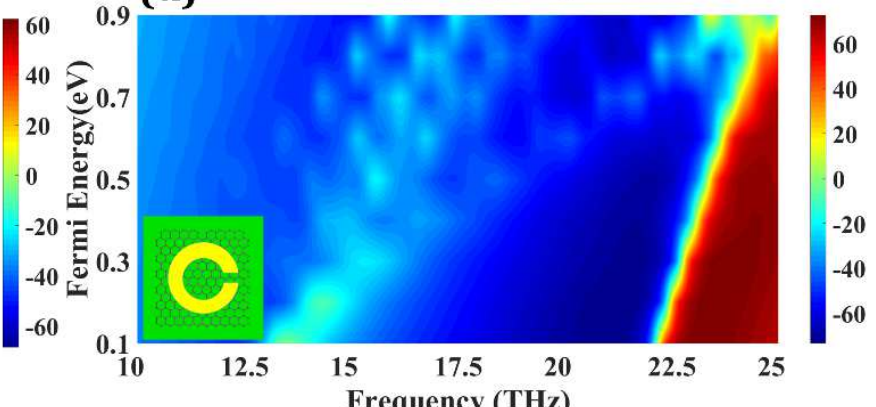

Calculated phase variation $\Phi_{x x}$ for the different values of fermi energy and frequency for X polarized condition. Values of $\Phi_{x x}$ for (a) RR, (b) SSRR, (c) TSRR and (d) SRR structures. Figure 5:

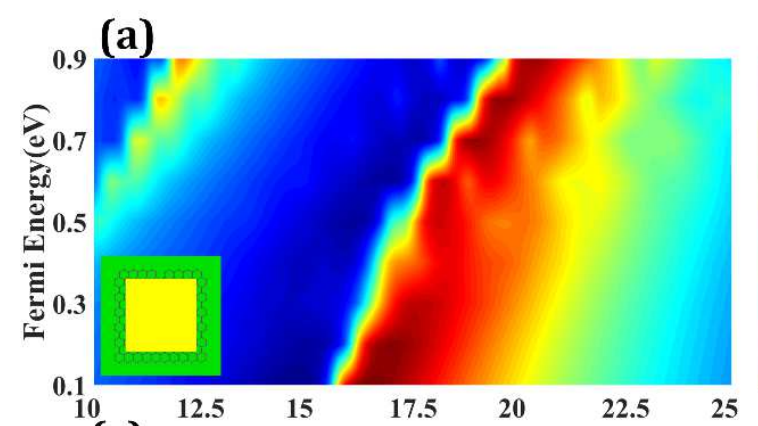

(c) Frequency $(\mathrm{THz})$
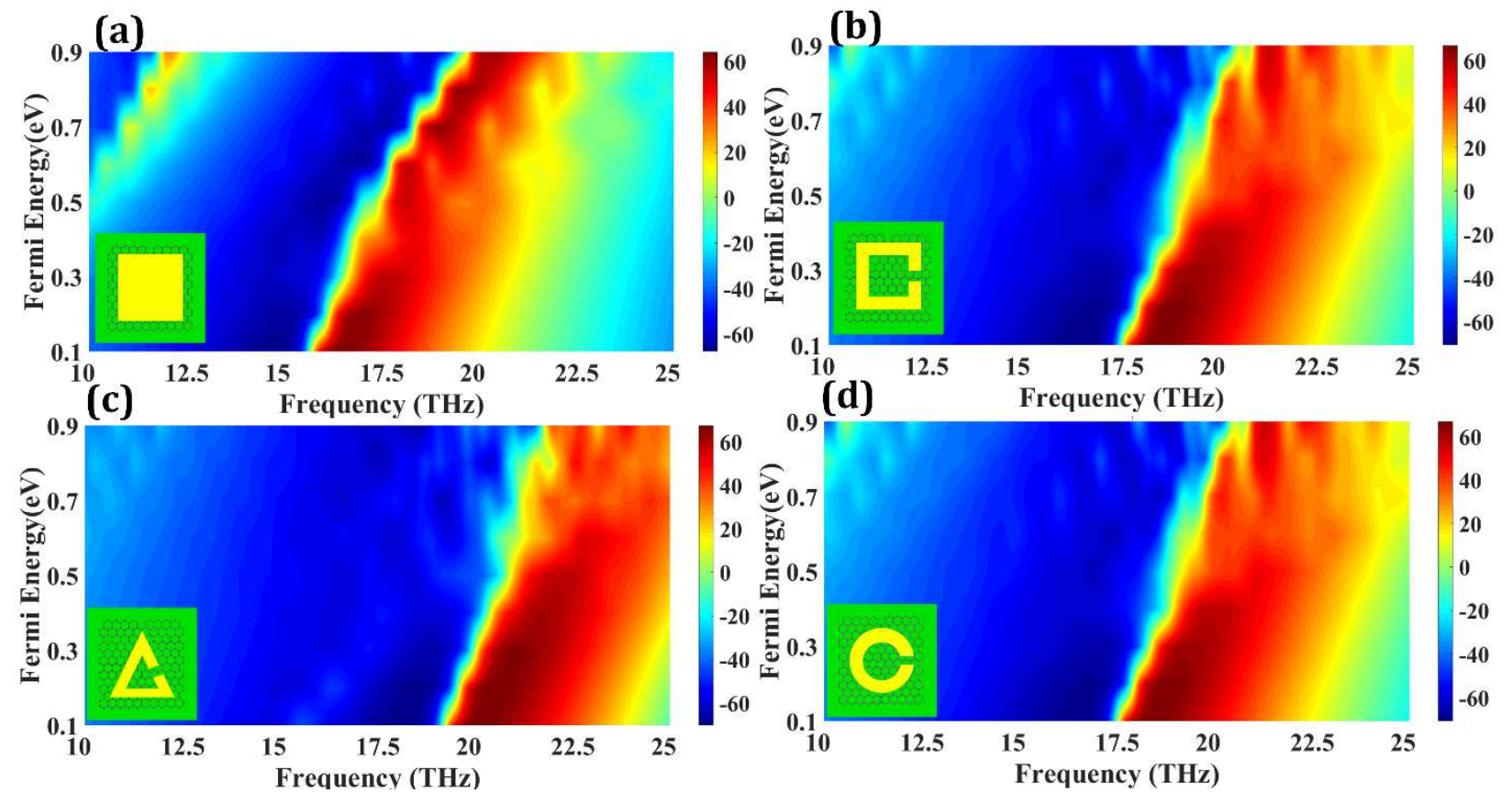

(d) $\begin{array}{rrr}12.5 & 15 \quad 17.5 & 20 \\ \quad & \text { Frequency }(\mathrm{THz})\end{array}$

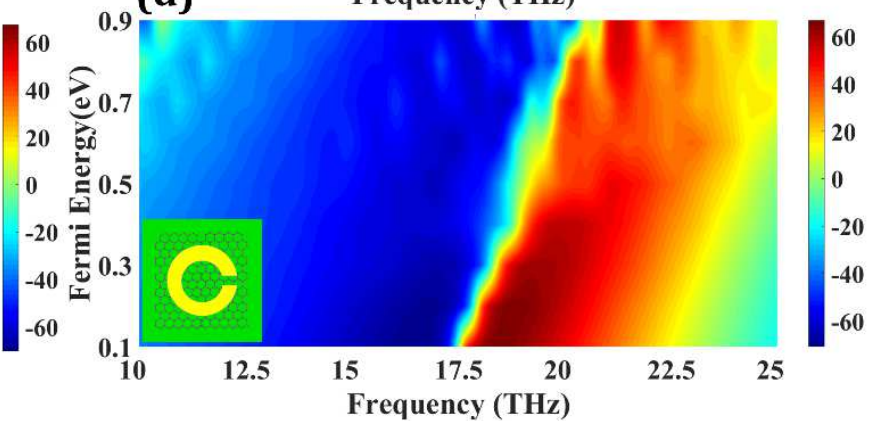

Calculated phase variation $\Phi_{y y}$ for the different values of Fermi energy and frequency for Y polarized condition. Values of $\Phi_{y y}$ for (a) RR, (b) SSRR, (c) TSRR and (d) SRR structures. Figure 6: 

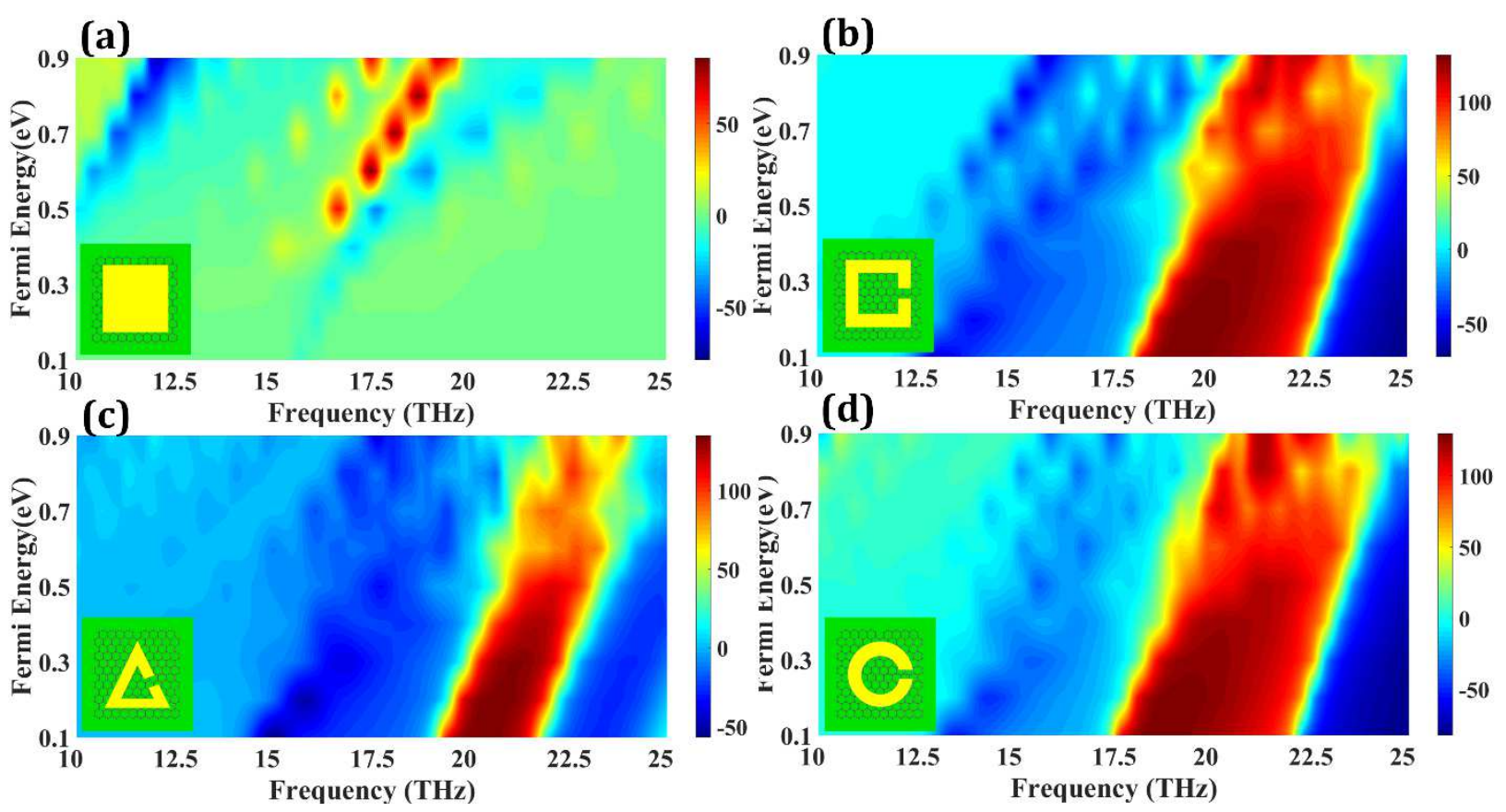

Calculated phase difference $\Delta \Phi$ for the different values of fermi energy and frequency for co polarization condition. Values of $\Delta \Phi$ for (a) RR, (b) SSRR, (c) TSRR and (d) SRR structures. 
Figure 7:

(a)

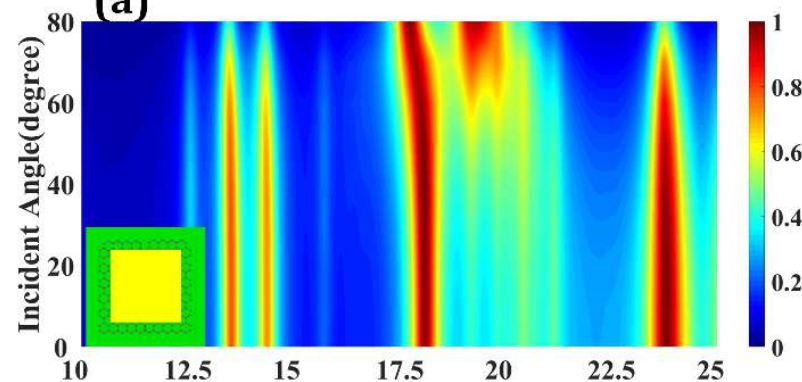

(c) Frequency (THz)

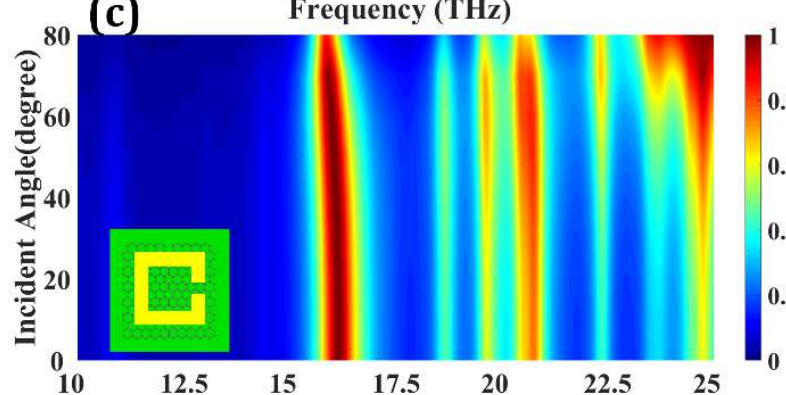

(e) Frequency $(\mathrm{THz})$
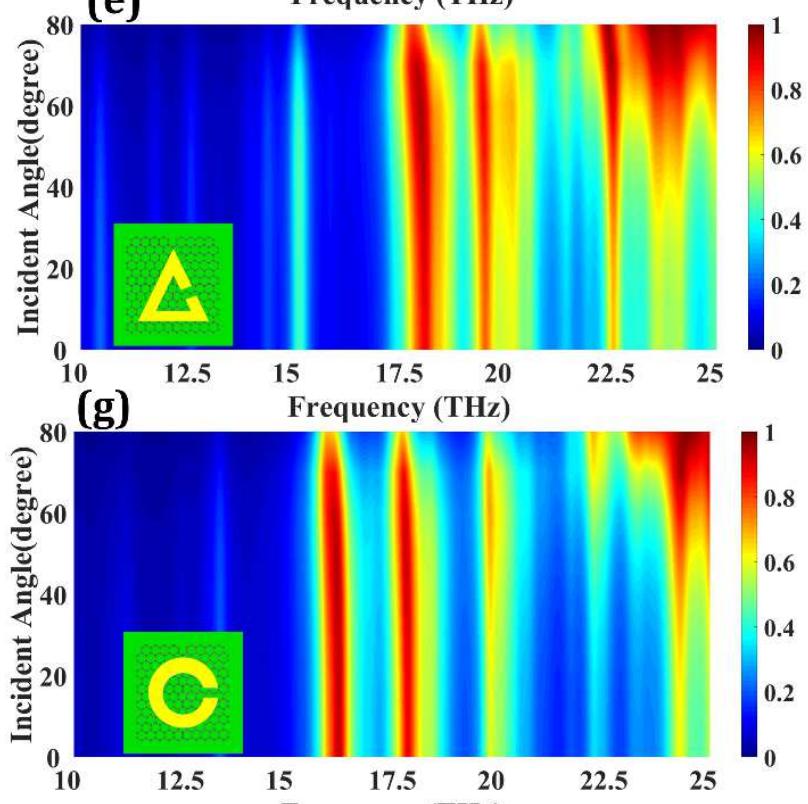

(b)

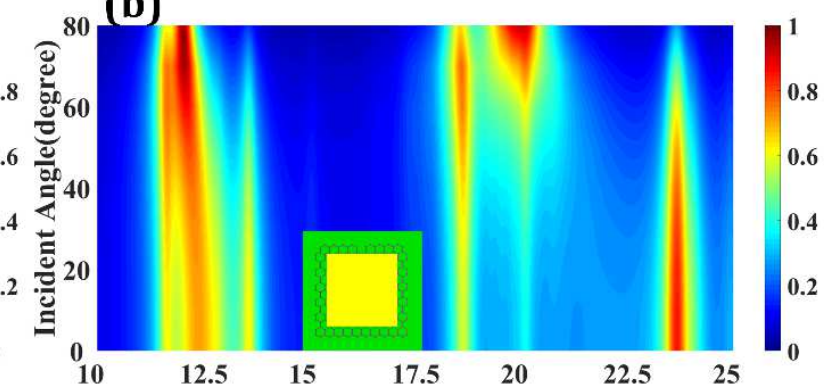

(d)

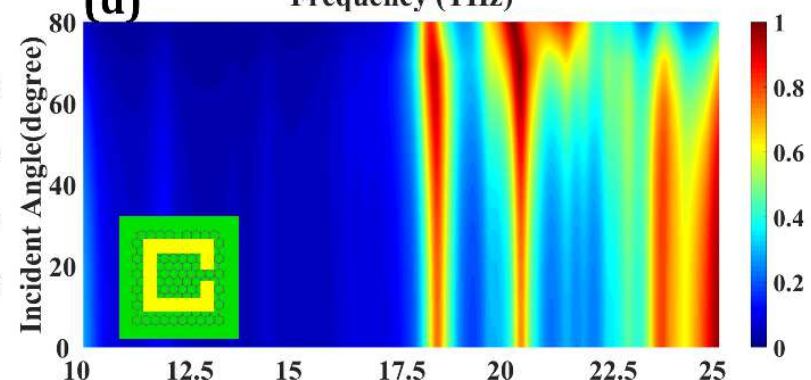

(f) Frequency (THz)

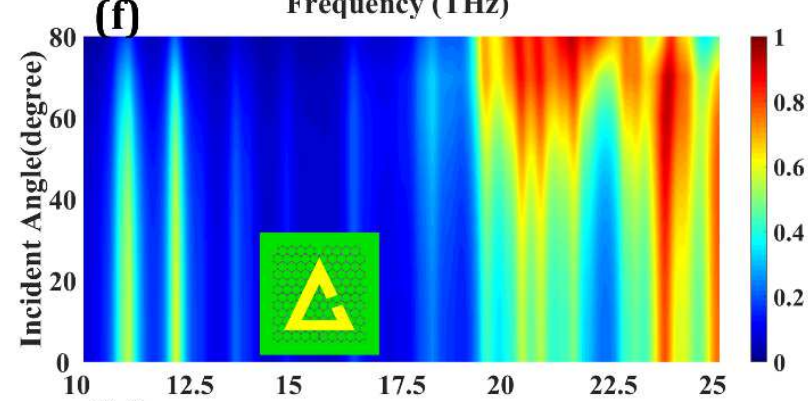

(h)

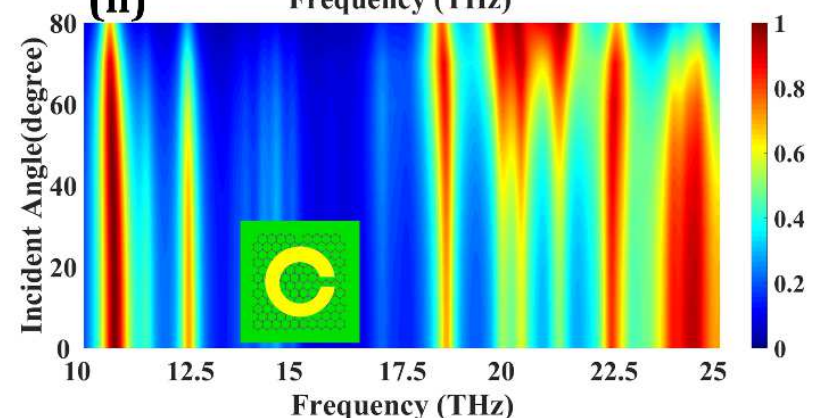

Variation in the reflectance coefficient for the wide-angle incident values at different incident polarization conditions. (a) $R_{x x}$ and (b) $R_{y y}$ for RR geometry. (c) $R_{x x}$ and (d) $R_{y y}$ for SSRR geometry. (e) $R_{x x}$ and (f) $R_{y y}$ for TSRR geometry. (g) $R_{x x}$ and (h) $R_{y y}$ SRR geometry. The wideangle incident response has been derived for the $0^{\circ}$ to $80^{\circ}$ of incident angle. 
Table 1: Parameter specification and values of Eq. $(1,2)$

\begin{tabular}{|l|l|l|}
\hline Parameters & Values & Specification \\
\hline$\omega$ & $3 \mathrm{THz}$ to $12 \mathrm{THz}$ & Radian frequency \\
\hline$\Gamma$ & $10^{-11}$ & Scattering rate \\
\hline $\mathrm{h}$ & $6.582119569 \times 10^{-16} \mathrm{eV} . \mathrm{s}$ & Reduced plank constant \\
\hline$k_{\mathrm{B}}$ & $\begin{array}{l}8.617333262145 \times 10^{-5} \mathrm{eV} . \mathrm{K}^{-} \\
1\end{array}$ & Boltzmann constant \\
\hline$E_{f}$ & $0.1 \mathrm{eV}$ to $0.9 \mathrm{eV}$ & Fermi energy \\
\hline$\tau^{-1}$ & $10^{-13} \mathrm{~s}$ & Electron relaxation time \\
\hline $\mathrm{T}$ & $300 \mathrm{~K}$ & Temperature \\
\hline
\end{tabular}

Table 2: Comparative analysis of the proposed structure with already available designs.

\begin{tabular}{|c|c|c|c|c|c|c|}
\hline Reference & $\begin{array}{c}\text { Unit Cell } \\
(\mathrm{L}, \mathrm{W}, \mathrm{H}) \mu \mathrm{m}^{3}\end{array}$ & $\begin{array}{c}\text { Constitutive } \\
\text { Material }\end{array}$ & $\begin{array}{l}\text { Maximum } \\
\text { Reflectance }\end{array}$ & $\begin{array}{c}\text { Graphene } \\
\text { layers }\end{array}$ & $\begin{array}{c}\text { Frequency } \\
\text { Band } \\
(\mathrm{THz})\end{array}$ & $\begin{array}{l}\text { Angular } \\
\text { Stability } \\
\text { (degree) }\end{array}$ \\
\hline This work & $(7.6,7.6,2.07)$ & S-G-Au & $>98 \%$ & Single & $10-25$ & $85^{\circ}$ \\
\hline$[35]$ & $(16,16,25)$ & $\mathrm{G}-\mathrm{Au}$ & $88 \%$ & Multiple & $0.6-2.6$ & $40^{\circ}$ \\
\hline [36] & $(120,120,59)$ & G-Au & $60 \%$ & Single & 0.4-1 & NA \\
\hline [37] & $(10,10,36)$ & $\mathrm{G}$ & $40 \%$ & Single & $1.23-2.73$ & NA \\
\hline [38] & $(100,100,26)$ & $\mathrm{M}$ & $30 \%$ & Multiple & $0.76-1.48$ & $40^{\circ}$ \\
\hline [39] & $(15,15,19)$ & $G$ & $75 \%$ & Multiple & $1.65-4.35$ & NA \\
\hline [40] & $(32,32,18)$ & $\mathrm{G}$ & $40 \%$ & Single & $2-4$ & NA \\
\hline [40] & $(32,32,18)$ & $\mathrm{G}$ & $40 \%$ & Single & $2-4$ & NA \\
\hline [41] & $(4,4,40.25)$ & G-S & $25 \%$ & Single & $1-4$ & NA \\
\hline [42] & $(70,70,25)$ & $\mathrm{TN}$ & $40 \%$ & Single & $1.17-2.99$ & $45^{\circ}$ \\
\hline [43] & $(200,200,9)$ & $\mathrm{M}$ & $50 \%$ & Single & $1.6-5$ & $70^{\circ}$ \\
\hline [44] & $(105,105,125)$ & $\mathrm{M}$ & $20 \%$ & Multiple & $0.7-2.5$ & $60^{\circ}$ \\
\hline
\end{tabular}

